\title{
Experimental Study on the Alteration of Cooling Effectivity Through Excitation-Frequency Variation Within an Impingement Jet Array with Side-Wall Induced Crossflow
}

\author{
Arne Berthold and Frank Haucke
}

\begin{abstract}
The influence of in-phase variation of the excitation frequency of a 7 by 7 impinging jet array between $f=0$ and $1000 \mathrm{~Hz}$ on the cooling effectivity is investigated experimentally. Liquid crystal thermography is employed to measure a 2-dimensional wall-temperature distribution, which is used to calculate the local Nusselt numbers and evaluate the global and local heat transfer. The cooling effectivity of the dynamic approach is determined by comparison with corresponding steady blowing conditions. The results show that the use of a specific excitation frequency allows a global cooling effectivity increase of more than $50 \%$.
\end{abstract}

Keywords Heat transfer $\cdot$ Experimental $\cdot$ Internal cooling Dynamic impingement cooling $\cdot$ Crossflow $\cdot$ Pulsed blowing

\section{Introduction}

The Collaborative Research Center "SFB 1029" is focused on the overall efficiency enhancement of gas turbines. The classical way to improve the overall gas turbine efficiency is to increase the turbine inlet temperature as well as the turbine pressure ratio. These specific approaches have been implemented over the last decades. Therefore, until today turbine inlet temperature has increased constantly, but also the divergence to the maximum permitted material temperatures. Due to the increase in turbine inlet temperature, convective cooling concepts become more relevant for the design of modern gas turbines or aero engines. Thereby, modern turbine cooling strategies are based on the combination of high-temperature proofed super alloys or

\footnotetext{
A. Berthold $(\varangle) \cdot$ F. Haucke

Department of Aeronautics and Astronautics, Chair of Aerodynamics, Technische Universität Berlin, Marchstr. 12-14, 10587 Berlin, Germany e-mail: Arne.Berthold@tu-berlin.de 
ceramic materials, film cooling for local hot-gas temperatures higher than $1600 \mathrm{~K}$ and internal cooling concepts for temperatures between 1300 and $1600 \mathrm{~K}$ [1]. Resulting from the steady increase in temperature modern turbines are already operating at the temperature limit of the coating materials. Thus, the logical conclusion is that a further increase in overall turbine efficiency is possible if the efficiency of the cooling concepts is improved as well. A second approach to increase the overall turbine efficiency is to switch the constant-pressure combustion to a constant-volume combustion. Following this approach, the SFB 1029 focuses on classical pulsed detonation [2] and on a new shockless explosion concept [3, 4]. In both cases the combustion process is highly unsteady and induces periodic pressure and temperature changes, which influence the flow characteristics of all gas turbine components. On the one hand, this leads to a higher turbine pressure ratio combined with an increased turbine inlet temperature. On the other hand, turbine inlet conditions can be kept constant, which leads to a reduced number of compressor stages due to the increase in pressure ratio through the combustion process. However, the cooling of the turbine blade is an important limiting parameter and therefore it is necessary to develop improved cooling mechanisms. One starting point for the improvement is the already implemented internal impingement cooling. Steady impinging jets feature high local heat-transfer coefficients compared to standard convective cooling inside turbine blades. After impinging on the hot inner surface, the cooling air mass flow is directed to the trailing edge and is discharged to the main hot gas flow. Thereby, upstream jets generate a cross flow that superimposes downstream impinging jets. The geometrical configuration, including nozzle diameter, nozzle distance, nozzle arrangement and impingement distance as well as Reynolds number of impinging jets, are important influencing parameters, which have been investigated by Florschuetz et al. [5, 6], Weigand and Spring [7] and Xing et al. [8]. To improve this well-established cooling mechanism, one part of the SFB is focused on research and development of dynamically forced impingement jet arrays. Due to the generation of strong vortex structures and their interactions with adjacent ones, the local convective heat transfer on the target surface can be enhanced. Thereby, the efficient exploitation of cooling air mass flow, typically originated from the high-pressure compressor, can be maximized. First experiments with a single forced impingement jet were performed by Liu und Vejrazka $[9,10]$. They stated that the forcing of the impingement jets can affect the heat transfer in the wall jet region while the heat transfer within the stagnation area is almost uninfluenced. Additionally, they pointed out that this result is dependent on the nozzle to impingement plate distance. An additional advancement is the mixing effect due to the interaction between jet and environment studied by Hofmann in 2007. The mixing can reduce the jet velocity as well as the heat transfer on the target plate, especially for large impingement distances. The smaller the impingement distances, the less is the mixing effect. The heat transfer can be enhanced if the Strouhal number is of the order of the turbulence magnitude. Thereby, a threshold Strouhal number of $S r_{D}=0.2$ was determined [11]. These results correspond with the findings of Gharib et al. from 1998 [12]. Gharib defines a formation number $t^{*}=\frac{\left(u_{p} \cdot t\right)}{d}$, which describes the generation of high-energy ring vortices in dependency of the exit 
velocity of a nozzle $u_{p}$, the process time $t$ and the nozzle diameter $d$. Janetzke [13] interpreted the formation number as the reciprocal of the Strouhal number. Therefore, the limits Gharib introduced to produce ring vortices with maximized size, vorticity and amplitude can be linked to the work of Herwig et al. [14], Middleberg et al. [15] and Janetzke et al. [13]. They describe the production of very strong vortices using square pulses. This enforces periodically strong local and temporal velocity gradients and thus maximizes local convective heat transfer. Influenced by the actuator characteristic there is a dependency between the enhancement of local Nusselt number and the combination of Strouhal number and amplitude. The possible combinations of geometrical and dynamic parameters is very large. The characteristics of an actuation system plays an important role as well. Thereby, the impact of dynamically forced impinging jets on the local heat transfer in the stagnation and wall jet zone needs to be studied in detail.

The present study is focused on the experimental investigation of the local convective heat transfer of an array of 7 by 7 dynamically forced impinging jets with superimposed crossflow. In particular, the study investigates the local convective heat transfer as a function of the excitation frequency and the impingement distance as well as the Reynolds number. The mayor focus thereby is the maximization of the local convective heat transfer, ergo the cooling effectivity inside of the turbine blade.

\section{Experimental Setup}

The basic experimental setup is schematically displayed in Fig. 1. Except for some minor changes the setup is comparable to previous work [16, 17]. The test rig allows a detailed flow field study under a 7 by 7 impingement jet array on a flat plate with superimposed crossflow. In this setup the crossflow is induced by side walls, which channels the accumulated mass flow from all nozzles towards one exit direction. Thereby, the normalized impingement distance $H / D$ (normalized by nozzle Diameter $D$ ) is defined through the height of the crossflow frame. Consequentially, the variation of the impingement distance implies the changing of the crossflow frame.

The nozzles inside of the cooling array are equivalent to the work conducted by Janetzke [13] and consist of a simple drill hole with an exit diameter of $D=12 \mathrm{~mm}$. The normalized spacing between two nozzles in every direction is $S / D=5$. The length over diameter ratio for each nozzle is $L / D=2.5$. In total the nozzle plate is equipped with 49 individual nozzles, which have an inline arrangement consisting of seven rows in each line.

Seven mass flow control units in cooperation with an in-house compressed air system are providing the required amount of air mass flow with an overall accuracy of $0.1-0.5 \%$. Each of the seven pressure support lines is feeding an individual air divider, which is supporting one row of nozzles transverse to the flow direction. To implement a dynamic forcing, each individual nozzle is equipped with a fast switching solenoid valve. The standard valve parameters are defined as: maximum normalized volume flow rate: $V_{N} \leq 160 l_{\mathrm{N}} / \mathrm{min}$ and maximum switching frequency: 
(a)

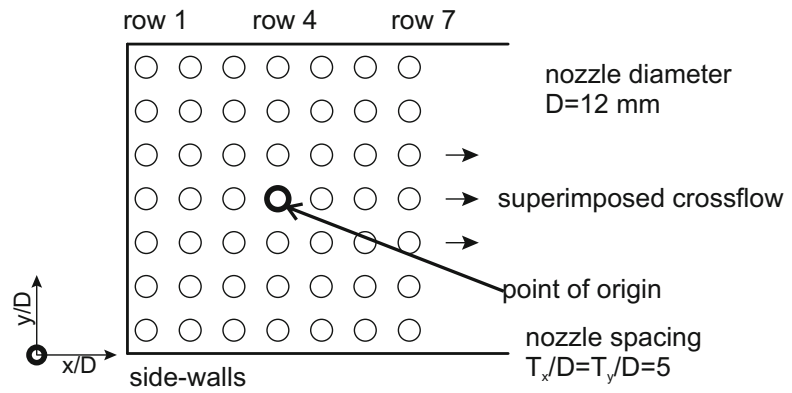

(b)

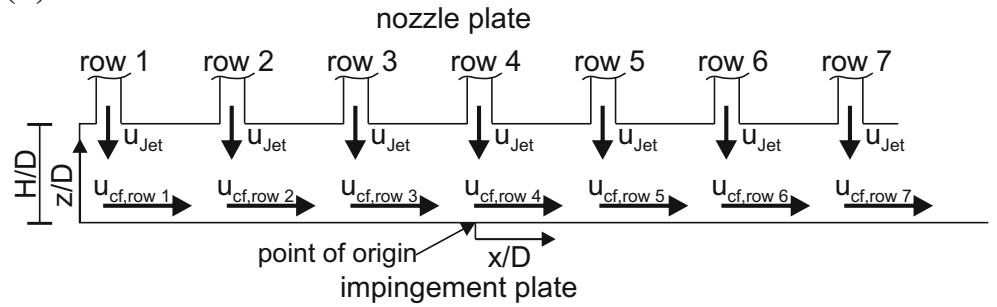

Fig. 1 a Schematic experimental setup b Schematic origin of $u_{c f} / U_{J e t}$

$f \leq 1000 \mathrm{~Hz}$. Since each solenoid valve can be controlled individually, a vast range of possible parameter set-ups can be investigated with this testing rig. The presented data is acquired for the entire frequency range of the solenoid valves. The frequency variation is performed for three impingement distances $(H / D=2,3,5)$ and three Reynolds numbers $\left(R e_{D}=3200,5200,7200\right)$ at each impingement distance. The superimposed crossflow for all experiments is generated by channelling the entire cooling massflow into one direction. Subsequently, the average crossflow velocity $U_{c} f$ is increased with every row of impingement nozzles until it reaches its maximum value behind the last row. This concept is comparable to the design of a turbine blade in which the cooling massflow is feeding the superimposed crossflow as well. Figure $1 \mathrm{~b}$ displays the schematic crossflow velocity increase $U_{c f, \text { row } 1 \ldots 7}$ while the exit velocity for each nozzle $u_{J e t}$ is kept constant. Due to the fact that the used cooling massflow, is equivalent to the crossflow massflow the quotient $\frac{U_{c f, r o w x}}{u_{\text {Jet }}}$ is linear increased with every row of nozzles while it stays constant for different Reynolds numbers at a specific impingement distance $H / D$. Variation of the impingement distance changes the cross section of the crossflow channel. As a result, the velocity quotient is inverse proportional to the impingement distance if the nozzle Reynolds number is kept constant. Table 1 displays the impingement nozzle position depending quotient for all tested cases.

The implemented measurement method for all the presented data is liquid crystal thermography (LCT). The thermochromic liquid crystal foil (Hallcrest "R35C5W") has a calibrated measurable temperature range within $T=35 \ldots 53^{\circ} \mathrm{C}$. The color 
Table 1 Impingement distance dependent increase of crossflow velocity inside of the array

\begin{tabular}{l|l|l|l|l|l|l|l}
\hline$H / D$ & $\begin{array}{l}\frac{u_{c f, \text { row } 1}}{U_{\text {Jet }}} \\
{[\%]}\end{array}$ & $\begin{array}{l}\frac{u_{c f, \text { row } 1}}{U_{\text {Jet }}} \\
{[\%]}\end{array}$ & $\frac{u_{c f, \text { row } 1}}{U_{\text {Jet }}}$ & $\begin{array}{l}\frac{u_{c f, \text { row } 1}}{U_{\text {Jet }}} \\
{[\%]}\end{array}$ & $\begin{array}{l}\frac{u_{c f, \text { row } 1}}{U_{\text {Jet }}} \\
{[\%]}\end{array}$ & $\begin{array}{l}\frac{u_{c f, \text { row } 1}}{U_{\text {Jet }}} \\
{[\%]}\end{array}$ & $\begin{array}{l}\frac{u_{c f, \text { row } 1}}{U_{\text {Jet }}} \\
{[\%]}\end{array}$ \\
\hline 2 & 7.9 & 15.8 & 23.7 & 31.6 & 39.5 & 47.4 & 55.3 \\
\hline 3 & 5.3 & 10.6 & 15.9 & 21.2 & 26.5 & 31.8 & 37.1 \\
\hline 5 & 3.2 & 6.4 & 9.6 & 12.8 & 16 & 19.2 & 22.4 \\
\hline
\end{tabular}

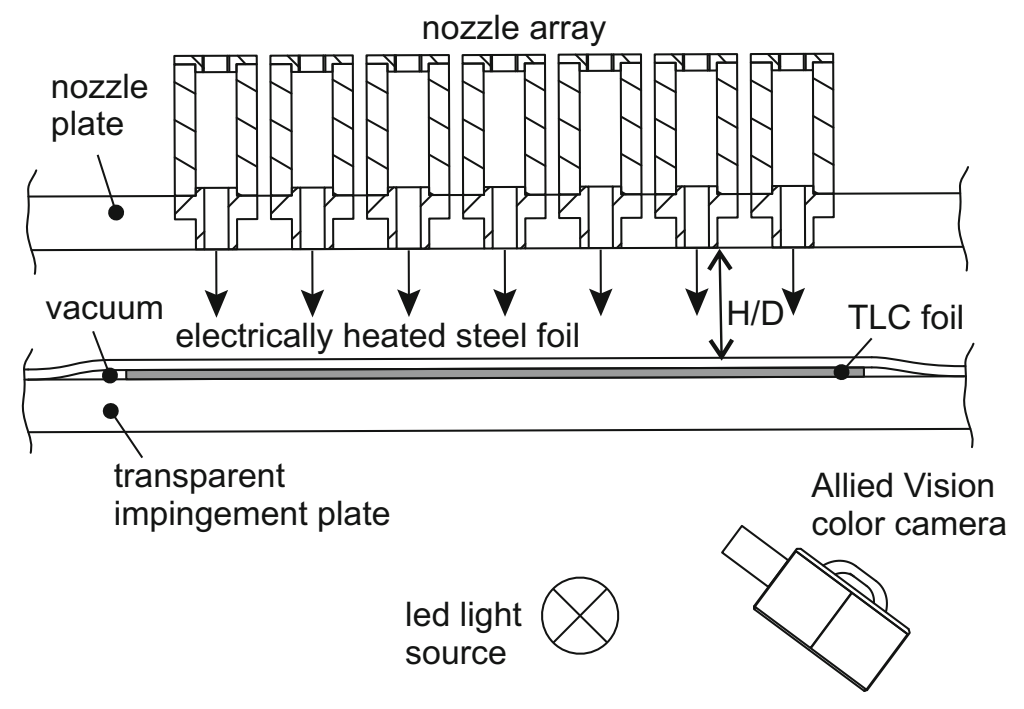

Fig. 2 Schematic setup of impingement plate

range starts with red (cold) and changes over green to blue and violet (hot). If the temperature range is exceeded, the TLC foil appears constantly black and thus temperature information is not analyzable. To minimize the measurement inaccuracy of the TLC foil, a color calibration is performed, which includes the simultaneous temperature depending acquisition of the color parameters hue, saturation and value (HSV). Given that the illumination for all measurements is kept constant, it is possible to determine a temperature band in which every temperature value has a unique combination of the three calibration parameters. Therefore, if the calibration is acquired with the necessary accuracy it is possible to reduce the overall uncertainty for the temperature depending color values to $\Delta T= \pm 0.1 \mathrm{~K}$.

Figure 2 schematically displays the construction of the impingement plate, which allows the LCT-measurement. The plate is a sandwich construction containing a thin steel foil $(600 \mathrm{~mm} \times 600 \mathrm{~mm} \times 0.05 \mathrm{~mm})$, which has the self-adhesive TLC foil attached to its rear side. The two layers are placed on a transparent glass plate $(1 \mathrm{~m}$ $\times 1 \mathrm{~m} \times 0.012 \mathrm{~m}$ ) and the edges are vacuum sealed in order to press the laminate together. The blank side of the steel foil is directed to the impinging jets, while the 
visible side of the TLC foil is oriented to the transparent impingement plate. The steel foil is connected to a power supply with a maximum electrical output of $P_{\max }=3700$ W. Controlling the electrical current, the steel foil can be heated continuously until a thermal equilibrium for the entire test chamber is obtained. Due to the resulting wall heat flux, the TLC foil is influenced thermally and a time averaged temperature depending color distribution can be measured. The electrical energy is adjusted for each operating point, which is defined by Reynolds number $R e_{D}$ and temperature range of the TLC foil. Depending on the resulting local wall temperatures, the liquid crystals reflect specific wave lengths of the light source through the glass plate back to a color camera. Through post processing it is possible to convert these RGBcolorspace values into HSV-colorspace values. After dewarping and further post processing of the raw images, a wall temperature distribution is extractable for further processing.

To determine the Nusselt number distribution on the impingement plate, it is assumed that the measured electrical power is equivalent to the emitted heat flux over the given heated area. In this consideration, the heat conduction into the adjacent wall structure as well as radiation effects are neglected. The measurement is performed when the entire system is in a state of thermal equilibrium. Hence, the wall heat flux is transferred completely into the cooling air mass flow of the impinging jets. The local Nusselt numbers can be calculated through the electrical Power $P$ in relation to the heated area $A_{\text {heat }}$. The balance between wall and nozzle temperature $\left(T_{W}-T_{D}\right)$ (static nozzle temperature), and the thermal conductivity of air $\lambda_{\text {air }}$ as presented in Eq. 1. To acquire the static nozzle temperature, the total nozzle temperature $T_{0}$ is measured inside of the nozzle aperture. Due to the low Mach number $(M a=0.03)$ the relation between the total and the static temperature is around $T_{0} / T_{D} \approx 1$. Hence, the measured total temperature value can be estimated as static nozzle temperature $T_{D}$.

$$
N u_{D}=\frac{\dot{q}}{T_{W}-T_{D}} \cdot \frac{D}{\lambda_{\text {air }}}=\frac{P}{A_{\text {heat }} \cdot\left(T_{W}-T_{D}\right)} \cdot \frac{D}{\lambda_{\text {air }}}
$$

If all measurement uncertainties are considered, then the overall uncertainty of the Nusselt number can be determined as $\delta N u_{D} / N u_{D}=3-8 \%$. Reproducibility studies on the presented experimental setup showed a maximal random uncertainty of below $3 \%$.

\section{Results}

Figure 3a presents the calculation basis of the most important quantities, which can be calculated from the 2-dimensional Nusselt number distribution. $\overline{N u}$ represents the mean global Nusselt number of the entire 2-dimensional field, which is used to categorize the general influence of the variable parameters. The second quantity is the crossflow oriented spatial development of the Nusselt number $\overline{N u}_{x}$. This value is the average value normal to the crossflow in $\mathrm{y}$-direction on every position $\mathrm{x}$. For 

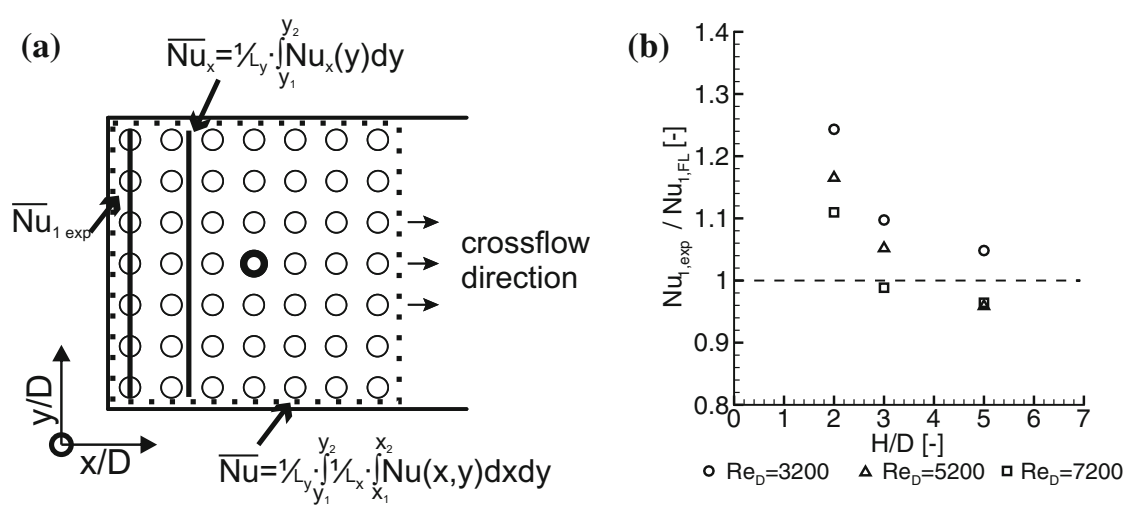

Fig. 3 a Schematic display of the calculation base used for the determination of the major quantities b Comparison computed data $\overline{N u}_{1, F L}$ to experimentally acquired data $\overline{N u}_{1, \exp }$

the first focus of the study, the spatial development in the center of the first upstream span wise row of nozzles is calculated and designated $\overline{N u}_{1, \exp }$. The value can be compared to an estimation presented by Florschuetz in 1981 [6]. Equation (2) can be employed to create a theoretical database, which is used to compare the experimental data with.

$$
\overline{N u}_{1, F L}=\left(x_{n} / D\right)^{-0.554} \cdot\left(y_{n} / D\right)^{-0.422} \cdot(z / D)^{0.068} \cdot \operatorname{Re}_{D}^{0.727} \cdot \operatorname{Pr}^{1 / 3}
$$

This equation includes crossflow, which is induced by side walls such as depicted in Fig. 1. Thereby, the crossflow component is a result of the jet nozzle Reynolds number and the geometry formed by the nozzle plate, the impingement surface and the side walls. Additionally, this equation is only valid if a steady blowing impingement jet is assumed. The computed heat transfer coefficients can be compared to the previously introduced value of $\overline{N u}_{1 \text { exp }}$ if all these requirements are implemented into the experimental setup. To allow such a comparison, every data set contains a steady blowing case to check if the acquired data is inside of the expected ratio presented by Florschuetz. Figure $3 \mathrm{~b}$ shows the ratio between the experimentally acquired data and the calculated $\overline{N u}_{1, F L}$ value for all tested impingement distances as well as nozzle Reynolds numbers.

For the impingement distances of $H / D=5$ it is apparent that the maximum deviation, of the quotient from the expected value, is $\Delta \overline{N u}_{1, \text { exp }} / \overline{N u}_{1, F L}= \pm 4.2 \%$. The deviation is slightly decreased as well if the nozzle Reynolds number is increased. A comparable nozzle Reynolds number dependent trend is apparent for an impingement distance of $H / D=3$, however, both extrema are intensified. Therefore, the maximum deviation is $9.6 \%$ and the minimum deviation is $-2.2 \%$. This performance is in accordance with Florschuetz's results, who stated an equation accuracy of $11 \%$ for $95 \%$ of his experimental correlations points. In case of the impingement distance of $H / D=2$ only the highest nozzle Reynolds number $R e_{D}=7200$ is $0.2 \%$ 
below the $11 \%$ deviation line. Again, a reduction of the nozzle Reynolds number increases the deviation up to $\Delta \overline{N u}_{1, \exp } / \overline{N u}_{1, F L}=16 \%$ for an nozzle Reynolds number of $R e_{D}=5200$ and $\Delta \overline{N u}_{1, \exp } / \overline{N u}_{1, F L}=24 \%$ for an nozzle Reynolds number of $R e_{D}=3200$. This result was to be expected because the used test rig operates at geometrical conditions, which are comparable to a set-up described as critical by Florschuetz. These set-ups tend to create bigger deviations from the estimation function if the impingement distance and the nozzle Reynolds number is decreased. In general, the acquired experimental data is in accordance with the results of Florschuetz.

After the determination and validation of the steady blowing cases, the second focus of the presented study is the determination of the heat transfer coefficients with respect to the excitation frequency, impingement distance and nozzle Reynolds number. Therefore, all impingement jets were phase averaged with an excitation frequency between $f=0 \ldots 1000 \mathrm{~Hz}$. The mean Nusselt number for all steady blowing cases $\overline{N u}_{0}$ as well as every individual dynamic forcing case $\overline{N u}$ over the entire flow field is calculated. The nozzle Reynolds number and impingement distance depending quotient $\overline{N u} / \overline{N u}_{0}$ in dependency of the excitation frequency is displayed in Fig. 4. It is apparent that the changeover from steady state blowing to dynamically forced blowing increases the global cooling effectivity $\overline{N u} / \overline{N u}_{0}$ for every test case at any tested frequency. Furthermore, it is evident that the general trend of all investigated nozzle Reynolds numbers for all impingement distances are quite similar. All these cases show an increased Nusselt number quotient in an excitation frequency band between $f=100 \mathrm{~Hz}$ and $f=200 \mathrm{~Hz}$. In case of the impingement distance of $H / D=2$ and $H / D=3$ this peak value is followed by a frequency band between $f=200 \mathrm{~Hz}$ and $f=500 \mathrm{~Hz}$, in which the value of the Nusselt number increase is nearly constant. A strong Nusselt number increase becomes observable, if the excitation frequency is increased above $f=500 \mathrm{~Hz}$. The increase continues until it reaches the global maximum at a frequency of $f=700 \mathrm{~Hz}$. After the maximum the Nusselt number starts to decrease with further increase of the excitation frequency until the minimum is reached at an excitation frequency of $f=1000 \mathrm{~Hz}$.

Additional findings need to be discussed, if the impingement distance of $H / D=5$ is included into the analysis. The first peak in Nusselt number is for a nozzle Reynolds number of $R e_{D}=7200$, like all other cases at lower impingement distances, located around $f=100 \mathrm{~Hz}$, while both smaller nozzle Reynolds numbers show the first peak at a frequency around $f=200 \mathrm{~Hz}$. Additionally, the general trend inside of the frequency band between $f=300 \mathrm{~Hz}$ and $f=500 \mathrm{~Hz}$ is changed from a nearly constant development $(H / D=2$ and $H / D=3)$ to a monotonically increasing one for all nozzle Reynolds numbers. Beginning at a frequency of $f=500 \mathrm{~Hz}$ the inclination is strongly increased until a global maximum is reached at a frequency of $f=700 \mathrm{~Hz}$. Following the global maximum, the development of the Nusselt number is equivalent to previously discussed cases.

After the general determination of the global Nusselt number development, it is interesting to look at the maximum possible gain in global Nusselt number $\overline{N u} / \overline{N u}_{0}$ 


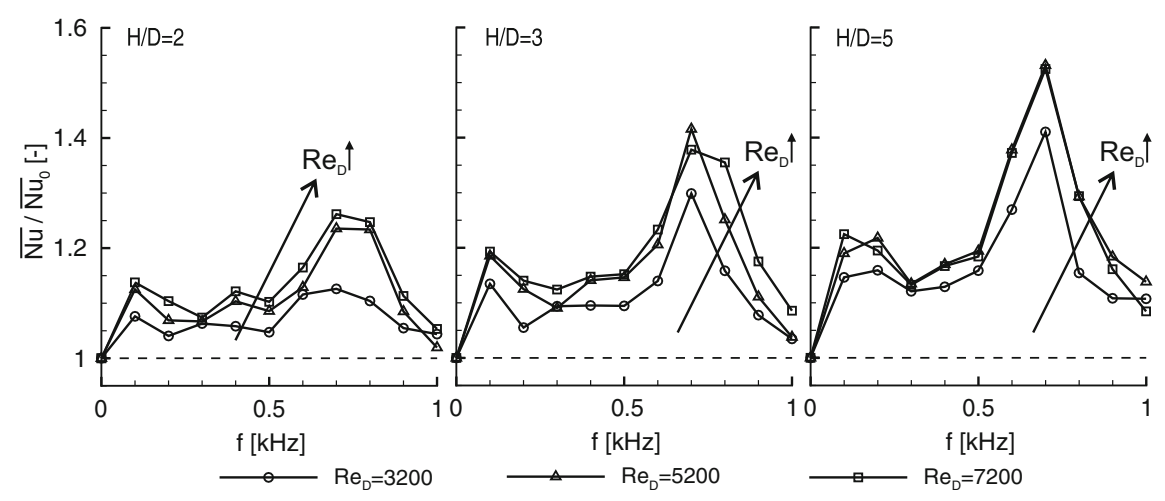

Fig. 4 Global development of the cooling in relation to excitation frequency, nozzle Reynolds number and impingement distance

Table 2 Percental increase in global Nusselt number through dynamic forcing compared to steady blowing

\begin{tabular}{l|l|l|l}
\hline$R e_{D}[-]$ & $\Delta \overline{N u}_{\max , H / D=2[\%]}$ & $\Delta \overline{N u}_{\max , H / D=3}[\%]$ & $\Delta \overline{N u}_{\max , H / D=5[\%]}$ \\
\hline 3200 & 12 & 29 & 40 \\
\hline 5200 & 23 & 41 & 52 \\
\hline 7200 & 26 & 37 & 52 \\
\hline
\end{tabular}

in relation to the impingement distance. For an nozzle Reynolds number of $R e_{D}=$ 7200 the global Nusselt number maximum at an impingement distance of $H / D=2$ is $\overline{N u} / \overline{N u_{0}}=1.26$, which equals an increase in cooling effectivity of $\Delta \overline{N u}_{\text {max }}=$ $26 \%$ compared to steady blowing. For an impingement distance of $H / D=3$ the cooling effectivity at the same frequency is increased by $\Delta \overline{N u}_{\max }=37 \%$ and for an impingement distance of $H / D=5$ the cooling effectivity is $\Delta \overline{N u}_{\max }=52 \%$ higher than for steady blowing. The same development is evident for the lower nozzle Reynolds numbers as well (see Table 2). Two trends are observable if all values are considered. The first one is that the maximum gain in cooling effectivity is increased with the impingement distance. A physical explanation can be found in the mixing process of the impingement jets. A steady state jet interacts with the surrounding fluid. Therefore, if the impingement distance is increased, the interaction length is increased as well. This leads to an increased mixing of the cooling fluid with the hotter surrounding fluid, which rises the resulting coolant temperature and therefore, reduces the cooling effect.

In case of dynamically forced impingement jets an approximated temporal square wave signal of the jet velocity can be assumed. The duty cycle is a direct driving parameter for estimating the peak velocity. Thereby, it equals the opening time of the fast switching valves during one oscillation period. For the present case, using 
a duty cycle of $D C=50 \%$, the peak nozzle Reynolds number $\operatorname{Re}_{D}$ is two times higher than the mean or steady blowing nozzle Reynolds number.

$$
\hat{R} e_{D}=\frac{R e_{D}}{D C}=2 \cdot R e_{D}
$$

As a result, the time averaged velocity for both cases is the same if the cooling air mass flow is kept constant. However corresponding to the equation the unsteady velocity distribution shows a doubling of the peak velocity in case of pulsed blowing. The doubling is a consequence of the bisected time in which the entire fluid has to be ejected through the nozzle. In this context strong fluctuating vortex structures can be generated, which are able to perculate the crossflow with a decreased level of interaction [16]. Therefore, the vortex rings can transport more cooling fluid directly to the impingement plate to increase the cooling effectivity. Interesting at this point is that for all investigated impingement distances and nozzle Reynolds numbers the maximum increase in cooling effectivity can be reached at an excitation frequency of $f=700 \mathrm{~Hz}$, which is in a frequency band determined by Janetzke [13] to produce mono frequent vortex rings. To determine the exact physical reason for this specific frequency, additional studies with focus on the physical differences inside of the flow field for different excitation frequencies will be conducted.

The second interesting characteristic is the nozzle Reynolds number influence on the global Nusselt number at a fixed impingement distance. In case of an impingement distance $H / D=2$ the potential gain in global cooling effectivity is steadily increased with the nozzle Reynolds number. If the maximum value at $f=700 \mathrm{~Hz}$ is used as reference, then the cooling effectivity increase between $R e_{D}=3200$ and $R e_{D}=5200$ is around $11 \%$ while the increase between $R e_{D}=5200$ and $R e_{D}=7200$ is only around $3 \%$. The behavior of the cooling effectivity deviation between the nozzle Reynolds numbers $R e_{D}=5200$ and $R e_{D}=7200$ is changed if the impingement distance is increased. While the increase between $R e_{D}=3200$ and $R e_{D}=5200$ is again around $12 \%$ for both greater impingement distances, it appears that the frequency depending global Nusselt number trends start to converge if the nozzle Reynolds number is increased above $R e_{D}=5200$. This converging process is also dependent on the impingement distance. Hence at an impingement distance of $H / D=3$ the trends for both higher nozzle Reynolds numbers are nearly superimposable. Smaller deviations are only noticeable in a frequency band between $f=700$ $\mathrm{Hz}$ and $f=1000 \mathrm{~Hz}$. The trends for both nozzle Reynolds numbers stays superimposable up to a frequency around $f=1000 \mathrm{~Hz}$ if the impingement distance is even further increased up to $H / D=5$. This result implies that the gain in cooling effectivity through dynamic forcing of the impingement jets is limited to a threshold at an impingement distance dependent nozzle Reynolds number. If this threshold nozzle Reynolds number is surpassed, then there seems to be a plateau in the frequency depending cooling effectivity increase.

In addition to the global development in cooling effectivity, it is commendable to look at the local distribution of the cooling effectivity in crossflow direction, to investigate where the frequency depending increase in cooling effectivity is generated. 


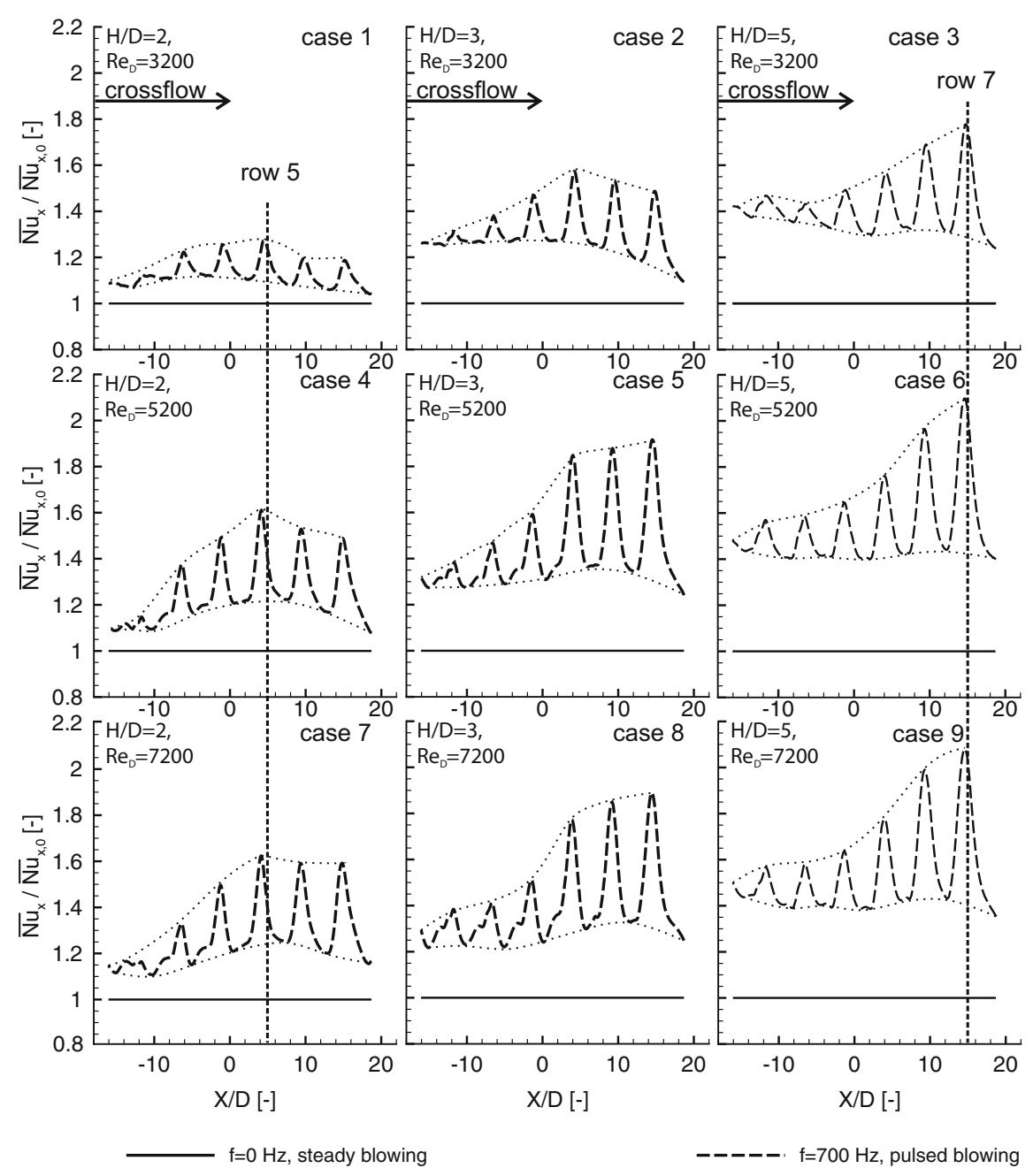

Fig. 5 Spatial distribution of Nusselt number in relation to the impingement distance and nozzle Reynolds number at $\mathrm{f}=700 \mathrm{~Hz}$

Therefore, it is necessary to analyze the crossflow oriented spatial development $(x / D)$ of the Nusselt number $\left(\overline{N u}_{x}\right)$. The distribution for steady blowing $(f=0 \mathrm{~Hz})$ shows a crossflow-oriented reduction of the cooling effectivity, which is increased with the crossflow velocity and, therefore, with the normalized coordinate $(x / D)$. This basic development is comparable to previously published data, which is addressing the specific phenomenon in detail [17]. A way to determine the influence of the excitation frequency on the local cooling effectivity is to normalize the dynamically forced spatial developments with the appropriate spatial development of the steady

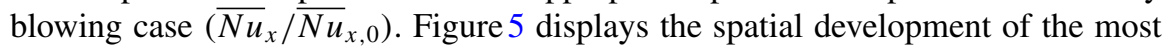


effective excitation frequency $(f=700 \mathrm{~Hz}$ ) for all investigated impingement distances and nozzle Reynolds numbers. It is evident that for a fixed nozzle Reynolds number both the lowest spatial cooling effectivity as well as the highest cooling effectivity is constantly raised if the impingement distance is increased. In addition to the raise of the levels, it is noticeable that the range between the low level cooling effectivity and the maximum value of local cooling effectivity is increased with the impingement distance as well. Furthermore, it is noticeable that the location of the maximum increase in cooling effectivity is locally shifted with the impingement distance. For the impingement distance of $H / D=2$ the global maximum is slightly upstream the fifth row of nozzles (see Fig. 2) while at an impingement distance of $H / D=5$ the maximum is located slightly upstream the last row of nozzles. A possible reason for this particular behaviour is that with the increase of the impingement distance at a fixed nozzle Reynolds number the crossflow velocity is decreased due to the enlargement of the cross-sectional-area. As a result, the interaction between the generated vortex rings and the resulting crossflow is reduced, which means that more uninfluenced cooling fluid is transported to the impingement plate. In contrast to the similar trends at a fixed nozzle Reynolds number the behaviour of the local Nusselt numbers is quite dissimilar if the impingement distance is kept constant for different nozzle Reynolds numbers. So at a impingement distance of $H / D=5$ the raise of the nozzle Reynolds number increases the local cooling effectivity (see case 3 and case 9). The general trend of the local cooling effectivity is similar in all three cases, therefore, the global maximum of the cooling effectivity is around the last nozzle and the total value of the gain in cooling effectivity is increased over the downstream rows of nozzles. A slightly different behaviour is noticeable if the nozzle Reynolds number variation at an impingement distance of $H / D=2$ (case 1 , case 4 and case 7) is included into the analysis. Again, the increase of the nozzle Reynolds number increases the maximum gain in local cooling effectivity but now a nozzle Reynolds number dependent change in the local trends is visible. In case 1 the maximum gain in local cooling effectivity is slightly upstream the fifth row of nozzles and the downstream rows show a significant decrease in downstream local cooling effectivity. In case 4 the same trend is noticeable, however the reduction effect at the downstream rows is reduced. This particular trend is continued in case 7 . In this particular case the values of the local cooling effectivity maxima are nearly identical to the value of the global maximum. An equal development is also noticeable in the cases at an impingement distance of $H / D=3$. The local trend of case 2 is thereby qualitatively similar to case 4 , while case 5 equals case 7 . The most interesting scenario at this impingement distance is case 8 . This particular case shows the same qualitative trend of the local cooling effectivity as the previously discussed cases at an impingement distance of $H / D=5$. The comparative analysis of the nozzle Reynolds number dependent behaviours at fixed impingement distances implies that the increase of the nozzle Reynolds number increases the crossflow component. Given that the increased crossflow is increasing the mixing effects between the impingement jets and the surrounding fluid and, therefore, decreasing the local cooling effectivity. Resulting from that the potential gain in local cooling effectivity through dynamic forcing is increased. The change of the general trends between 
the impingement distances indicates that the ability of the vortex rings to transport cooling fluid through the crossflow seems to increase stronger than the dampening effects of the crossflow. Additionally, it seems that the nozzle Reynolds number, at which the spatial shift of the global maximum is observable, has a inverse trend to the impingement distance. Hence, to achieve the spatial shift at low impingement distances, the Reynolds number needs to be increased.

\section{Conclusion}

Experimental research was performed to assess the alteration of the cooling effectivity in dependency of the excitation frequency $(0 \ldots 1000 \mathrm{~Hz})$, Reynolds number $\left(R e_{D}=3200,5200,7200\right)$ and impingement distance $(H / D=2,3,5)$ within a 7 by 7 nozzle impingement jet array with side-wall induced crossflow. Liquid Chrystal Thermography was employed to measure the 2-dimensional wall temperature field, which was used to calculate a Nusselt number distribution. The mean global Nusselt numbers for the steady blowing cases are comparable to well established values from literature and thus the data integrity is confirmed.

The values of global and local cooling effectivity for all combinations of the test parameters were compared to appropriate steady blowing cases to evaluate the impact of dynamically forced impingement jets inside of an array with superimposed crossflow. The analysis of the global cooling effectivity demonstrates an increase for all parameter combinations if any excitation frequency is applied. More detailed results show that the basic influence of the excitation frequency inside of a frequency band between $f=500 \mathrm{~Hz}$ and $f=1000 \mathrm{~Hz}$ is identical for all tested cases. The trend is increasing until the maximum Nusselt number ratio $\overline{N u} / \overline{N u}_{0}$ and thus the maximum cooling effectivity is reached at a frequency of $f=700 \mathrm{~Hz}$. For the most efficient combination of testing parameters, an increase in cooling effectivity of $52 \%$ was discovered. Following the maximum, a continuously decrease in cooling effectivity is observable. For frequencies up to $f=500 \mathrm{~Hz}$ the impingement distances of $H / D=2$ and of $H / D=3$ display an approximately constant Nusselt number ratio, while the ratio for $H / D=5$ is slightly increasing in the same frequency band. All test cases present a first peak value in cooling effectivity in a frequency band between $f=100 \mathrm{~Hz}$ and $f=200 \mathrm{~Hz}$.

A detailed analysis of the spatial developments in case of the most efficient excitation-frequency revealed a dependency of the location of the maximum gain in cooling effectivity on the impingement distance and the nozzle Reynolds number. Hence, for smaller impingement distances the maximum gain in local cooling effectivity is slightly before the fifth row of nozzles while for higher impingement distances the maximum is located slightly before the last row of nozzle at the same nozzle Reynolds number. It is striking that the potential gain through dynamic forcing is increased if the nozzle Reynolds number is increased at a fixed impingement distance. Additionally, the location of the global cooling effectivity maximum is shifted from slightly upstream the fifth row of nozzles to the last row. The nozzle 
Reynolds number that is required for the position shift of the global cooling effectivity maximum is increased if the impingement distance is reduced.

Follow up studies will be performed to understand the physical mechanisms which are responsible for the improvement of the cooling effectivity at an excitation frequency of $f=700 \mathrm{~Hz}$ as well as to determine the physical behaviour of the dynamic impingement jets and the impingement distance depending alteration of the cooling effectivity.

Acknowledgements The authors gratefully acknowledge support by the Deutsche Forschungsgesellschaft (DFG) as part of the collaborative research centre SFB 1029 "Substantial efficiency increase in gas turbines through direct use of coupled unsteady combustion and flow dynamics" (project B03). Additionally the authors thankfully acknowledge the support of the student research assistants B.Sc. Burcu Ataseven, B.Sc. Lennart Rohlfs and B.Sc. Melik Keller during the measurement process.

\section{References}

1. Bräunling, W.J.G.: Flugzeugtriebwerke, vol. 3. Springer, Auflage (2009)

2. Gray, J., Moeck, J., Paschereit, C.: Non-reacting investigations of a pseudo-orifice for the purpose of enhanced deflagration to detonation transition. In: Roy, G.D., Frolov, S.M. (eds.), International Conference on Pulsating and Continuous Detonations, Torus Press (2014)

3. Bobusch, B., Berndt, P., Paschereit, C., Klein, R.: Shockless explosion combustion: an innovative way of efficient constant volume combustion in gas turbines. Combust. Sci. Technol. 186(10-11):1680Ü1689, 2014, ISSN 0010-2202 (2014)

4. Bobusch, B., Berndt, P., Paschereit, C., Klein, R.: Investigation of fluidic devices for mixing enhancement for the shockless explosion combustion process. Active Flow Combust. Control (2014), S. 281Ü297. Springer, 2015, ISBN 3319119664 (2014)

5. Florschuetz,L.W., Metzger, D.E., Takeuchi, D., Berry, R.: Multziple jet impingement heat transfer characteristic - experimental investigation of in-line and staggered arrays with crossflow. NASA-CR-3217. Arizona State University, Tempe, Department of Mechanicle Engineering (1980)

6. Florschuetz, L.W., Truman, C.R., Metzger, D.E.: Streamwise flow and heat transfer distributions for jet array impingement with crossflow. J. Heat Transf. 103, 337-342 (1981)

7. Weigand, B., Spring, S.: Multiple jet impingement—a review. Heat Transf. Res. 42(2), 101-142 (2010)

8. Xing, Y., Spring, S., Weigand, B.: Experimental and numerical investigation of heat transfer characteristics of inline and staggered arrays of impinging jets. J. Heat Transf. 132, 092201/1$11(2010)$

9. Liu, T., Sullivan, J.P.: Heat transfer and flow structures in an excited circular impingement jet. Int. J. Heat Mass Transf. 39, 3695-3706 (1996)

10. Vejrazka, J., Tihon, J., Marty, P., Sobolik, V.: Effect of an external excitation on the flow structure in a circular impinging jet. Phys. Fluids 17, 1051021-01-14 (2005)

11. Hofmann, H.M., Movileanu, D.L., Kind, M., Martin, H.: Influence of a pulsation on heat transfer and flow structure in submerged impinging jets. Int. J. Heat Mass Transf. 50, 3638-3648 (2007)

12. Gharib, M., Rambod, E., Shariff, K.: A universal time scale for vortex ring formation. J. Fluid Mech. 360, 121-140 (1998)

13. Janetzke, T.: Experimental investigations of flow field and heat transfer characteristics due to periodically pulsating impinging air jets. Heat Mass Transf. 45, 193-206 (2008)

14. Herwig, H., Middelberg, G.: The physics of unsteady jet impingement and its heat transfer performance. Acta Mech. 201, 171-184 (2008) 
15. Middelberg, G., Herwig, H.: Convective heat transfer under unsteady impinging jets: the effect of the shape of the unsteadiness. J. Heat Mass Transf. 45, 1519-1532 (2009)

16. Haucke, F., Nitsche, W., Peitsch, D.: Enhanced convective heat transfer due to dynamically forced impingement jet array. In: Proceedings of ASME Turbo Expo 2016, No. GT2016-57360 (2016)

17. Berthold, A., Haucke, F.: Experimental investigation of dynamically forced impingement cooling. In: Proceedings of ASME Turbo Expo 2017, Vol. 5A: Heat Transfe, ASME Turbo Expo 2017: Turbomachinery Technical Conference and Exposition (2017) 